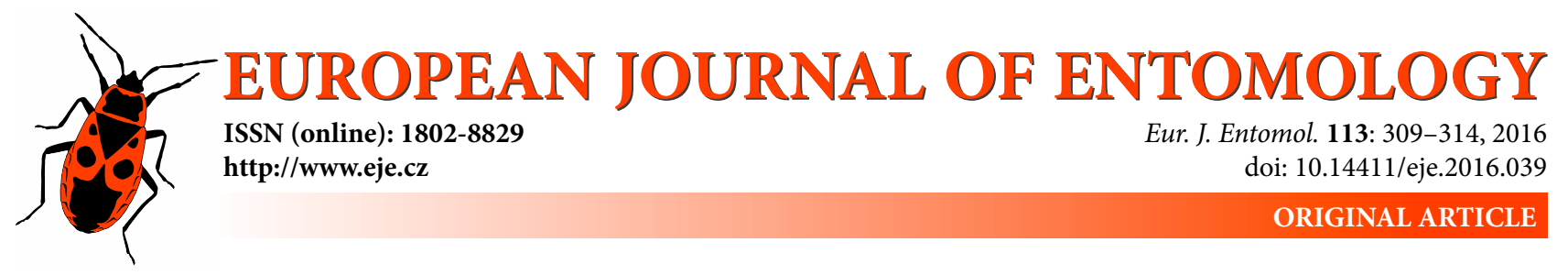

\title{
Reproductive status of Tribolium castaneum (Coleoptera: Tenebrionidae) affects its response to infection by Steinernema feltiae (Rhabditida: Steinernematidae)
}

\author{
Paulina KRAMARZ ${ }^{1}$, Dariusz MAŁEK ${ }^{1}$, MARIA GAWE ${ }^{1}$, SzYmon M. DROBNIAK ${ }^{1}$ and Joanna HOMA ${ }^{2}$ \\ ${ }^{1}$ Institute of Environmental Sciences, Jagiellonian University, Gronostajowa 7, 30-387 Krakow, Poland; \\ e-mails: paulina.kramarz@uj.edu.pl,dariusz.malek@uj.edu.pl, maria.gawel@uj.edu.pl, szymek.drobniak@uj.edu.pl \\ ${ }^{2}$ Institute of Zoology, Jagiellonian University, Gronostajowa 9, 30-387 Krakow, Poland; e-mail: joanna.homa@uj.edu.pl
}

Key words. Coleoptera, Tenebrionide, Tribolium castaneum, Rhabditida, Steinernematidae, Steinernema feltiae, phenoloxidase, sexual dimorphism, infection

\begin{abstract}
Gender-specific reproductive roles are important factors determining sexual dimorphism. Here, we investigate the effects of sex-based differences and reproductive status on the defence of Tribolium castaneum (Herbst, 1797) (Coleoptera: Tenebrionidae) against infection by Steinernema feltiae (Filipjev, 1934) (Rhabditida: Steinernematidae). Female and male beetles, either virgin or post-copulation, were exposed individually to nematodes. Individuals were then sampled every $12 \mathrm{~h}$, dissected, and checked for the presence of nematodes; we also measured their phenoloxidase (PO) activity. Reproductive status affected resistance to nematodes and PO activity as infected virgin individuals had a higher PO activity and lower mortality than reproducing individuals, with no differences between sexes. Mortality also increased with time, while PO activity did not change. Parasite load was related to reproductive status and sex, with reproducing females with the highest parasite loads in all treatments, and virgin males with more nematodes than sexually active males. Our results indicate that the costs of reproduction impair the immunological system of $T$. castaneum similarly in both sexes. It is possible, however, that other components of the immunological system that we did not measure, such as lysozyme activity, are impaired by infection with $S$. feltiae in a sex-specific way.
\end{abstract}

\section{INTRODUCTION}

Infection by parasites has serious fitness consequences for hosts, who may thus experience strong selective pressure to defend themselves (Schmid-Hempel, 2011). Therefore, a significant component of the life history strategy of most organisms is their investment in defence (SchmidHempel \& Ebert, 2003), often in the form of an immune system. However, the immune system is costly to both maintain and use (e.g., Kraaijeveld \& Godfray, 1997; Schmid-Hempel \& Ebert, 2003, Schmid-Hempel, 2011) and trade-offs are likely to arise that constrain its evolution.

Many studies indicate that there is a trade-off between immune function and reproductive effort (e.g. Adamo et al., 2001) and trade-offs between immunity and reproduction are a central concept in explanations of sexual selection (Zuk \& McKean, 1996; French et al., 2007). In fact, a reduction in immune function due to reproductive activity is documented for several species (Siva-Jothy et al., 1998; Adamo et al., 2001). There is, however, no evidence of reproductive activities resulting in a reduction in the immune response in two species of damselflies (Córdoba-Aguilar et al., 2011) or of a trade-off between reproduction and the response to the parasite (Serratia marcescens) in Acheta domesticus (Nava-Sánchez et al., 2015).

In promiscuous species, such the study species, Tribolium castaneum (Herbst, 1797) (Coleoptera: Tenebrionidae), sexes are expected to allocate resources in different ways. Male fitness is usually limited by the number of females fertilized, while female fitness is limited by the number of offspring produced. Males therefore increase fitness by increasing mating rates, while females gain fitness through increased longevity and resistance to parasites, a phenomenon known as Bateman's principle (Bateman, 1948; Rolff, 2002). Therefore, males are often more susceptible to parasites than females, in both vertebrates (e.g., Zuk, 1990; Poulin, 1996; Zuk \& McKean, 1996; Moore \& Wilson, 2002) and invertebrates (e.g., Gray, 1998; Wedekind \& Jakobsen, 1998; Adamo et al., 2001; Schwarzenbach et al., 2005; Córdoba-Aguilar \& Munguía-Steyer, 2013). In vertebrates this pattern is usually attributed to the immunosuppressive influence of testosterone (Alexander \& Stimson, 1988; Zuk, 1990). Insects lack testosterone, but instead, the production of juvenile hormone after copulation can down-regulate the expression of phenoloxidase (Rolff \& Siva-Jothy, 2002). 
Phenoloxidase is one of the most important immune responses in many insects. It is a key enzyme in the melanization cascade, which determines their resistance to different pathogens and is also involved in hardening of the shells of insects' eggs. Activity of this enzyme is often used to estimate immune function in insects (reviewed in Cerenius et al., 2008; González-Santoyo \& CórdobaAguilar, 2012). Phenoloxidase activity (and melanization in general) has profound fitness consequences in several pathogen-host systems, such as parasitoids and Drosophila melanogaster (Kraaijeveld \& Godfray, 1997) and Mnais costalis and Hoplorhynchus polyhamatus (Siva-Jothy et al., 2001). There is also evidence of trade-offs between phenoloxidase and other fitness traits such as development time and body mass (Cotter et al., 2004) or survival (Kraaijeveld \& Godfray, 1997). In contrast, reproductive activity in the damselflies, Argia anceps and Hetaerina americana, does not appear to have any influence on PO activity (Córdoba-Aguilar et al., 2011).

The red flour beetle (Tribolium castaneum) is a highly promiscuous species that is sexually dimorphic in size (Sokoloff, 1974) and in its immune response (Freitak et al., 2012). It is a major pest of stored food products, causing substantial losses to global grain harvests (Rossi et al., 2010). Entomopathogenic nematodes (EPN; Rhabditida: Steinernematidae and Heterorhabditidae) can be used as biological control agents against $T$. castaneum as they are commercially available (Georgis et al., 2006) and do not infest vertebrates (Bathon, 1996). They are obligate parasites of insects that go through a free-living dauer (infective) juvenile (IJ) stage. IJs invade their hosts via natural body openings, such as the mouth, anus and spiracles, and, once in the haemocoel, they release their bacterial symbionts (Xenorhabdus in Steinernema and Photorhabdus in Heterorhabditis), which kill the host within a few days (Hirao, 2010). Currently, infection by Steinernema feltiae (Filipjev, 1934) (Rhabditida: Steinernematidae), the parasite used in this study, is only lethal for larvae and pupae of $T$. castaneum; with the highest mortality recorded for adults ca. 40\% (Ramos-Rodríguez et al., 2006). This difference may indicate that adult individuals allocate more resources to immune defence against either the nematodes or their bacterial symbionts. Thus, the $S$. feltiae-T. castaneum system is ideal for investigating questions regarding sex-specific changes in resource allocation in hosts and the effect of these changes on host immunological responses. It also presents an opportunity to determine if hosts' reproductive efforts would change the effectiveness of nematodes as biological control factors.

Consequently, in this study we investigated the influence of sex and reproductive status (virgin versus reproducing) of $T$. castaneum beetles on their response to infection by $S$. feltiae. Towards this end, we measured both parasite load and phenoloxidase activity in infected and control beetles.

\section{MATERIALS AND METHODS}

\section{Experimental design}

The beetles in this study were kindly provided by B. Milutinović (see CR-01 in Milutinović et al., 2013). The strain is kept outbred at a constant temperature of $30^{\circ} \mathrm{C}$ (the "normal" temperature in this study) in constant darkness, and fed ad libitum on a medium composed of organic wheat flour and yeast ( $9: 1$ ratio). Tribolium castaneum beetles do not need additional water sources as they absorb humidity from the substrate (Sokoloff, 1974). The beetles were kept in plastic boxes with lids that had ventilation holes covered with steel mesh; the humidity in the culture was $70 \% \mathrm{RH}$. Experimental animals were reared under laboratory conditions for approximately 35 generations and kept outbred. A commercial strain of Steinernema feltie, e-nema, was kindly provided by R.-U. Ehlers.

In $T$. castaneum, parasite load can be measured by dissecting animals and phenoloxidase activity assessed only by using fresh samples. For this reason, these measurements were made on separate groups. Furthermore, a preliminary study indicated that, due to the small size of the beetles, the collection of haemolymph from numerous samples in a short time can only be done using whole animals.

We randomly chose 500 pupae of $T$. castaneum from the stock culture. Sex determination in this species is easiest at the pupal stage, so the experimental animals were divided into females and males as pupae and then allowed to mature in standard culture conditions. Five days after maturation, adults were divided into two groups: in the first, individual beetles were kept separate, while in the second group, one male was paired with one female and allowed to mate. After one week, the couples were separated, all beetles were weighed (Mettler Toledo Microbalance), and both virgin and reproducing individuals were divided into two groups: control and nematode-exposed. A dose of $60 \mathrm{IJs} /$ beetle was established in a preliminary study as high enough to ensure infection (i.e. allow IJs to enter into the beetle's body). The beetles were infected by placing them in Eppendorf tubes filled with $1 \mathrm{ml}$ of wet sand. The beetles were starved for the duration of the experiment, $48 \mathrm{~h}$, as in the preliminary study as no further infection by nematodes was recorded after that time. Infection was carried out at $25^{\circ} \mathrm{C}$, a temperature optimal for $S$. feltiae (Hirao et al., 2010) and still within the range of optimal temperatures for $T$. castaneum (Bucher, 2009).

From infected animals in each reproductive status group (female and male virgin, female and male post-reproduction), 20 individuals were sampled after 12, 24, 36 and $48 \mathrm{~h}$, and washed with Ringer's solution on a small sieve to remove any nematodes from the body surfaces. Then, 10 individuals were dissected and checked for the presence of nematodes, while the remaining 10 were frozen for the phenoloxidase measurements. Likewise, 10 of the control animals were sampled for phenoloxidase after the same time intervals and the remaining 10 were left in order to determine naturally occurring mortality.

\section{Phenoloxidase assay}

In total, 240 animals were checked for phenoloxidase. Our preliminary study showed that freezing did not change the measurements of phenoloxidase activity. Each animal was homogenized whole, diluted in $100 \mu \mathrm{l}$ of Ringer's solution and centrifuged at $4^{\circ} \mathrm{C}$ for $10 \mathrm{~min}$. Next, $10 \mu \mathrm{l}$ of each sample was placed in a $96-$ well plate. Four pseudo-replications were performed for each individual in order to detect artefactual readings during spectrophotometer measurements. We added $90 \mu$ of TRIS/ $\mathrm{Ca}^{2+}(0.1 \mathrm{M})$ and $10 \mu \mathrm{l}$ of L-DOPA ( $3 \mathrm{mg} \mathrm{ml}^{-1}$ in distilled water, Sigma-Aldrich Co., St. Louis, MO, USA) to each well, then incubated the samples in darkness at room temperature. Spectrophotometer measurements (wavelength $490 \mathrm{~nm}$, micro ELISA Reader Expert Plus, ASYS Hitach GmbH, Austria) were made after 5, 10, 15 and 30 min to estimate when phenoloxidase activity stabilized. For all measurements, phenoloxidase values stopped changing after 30 


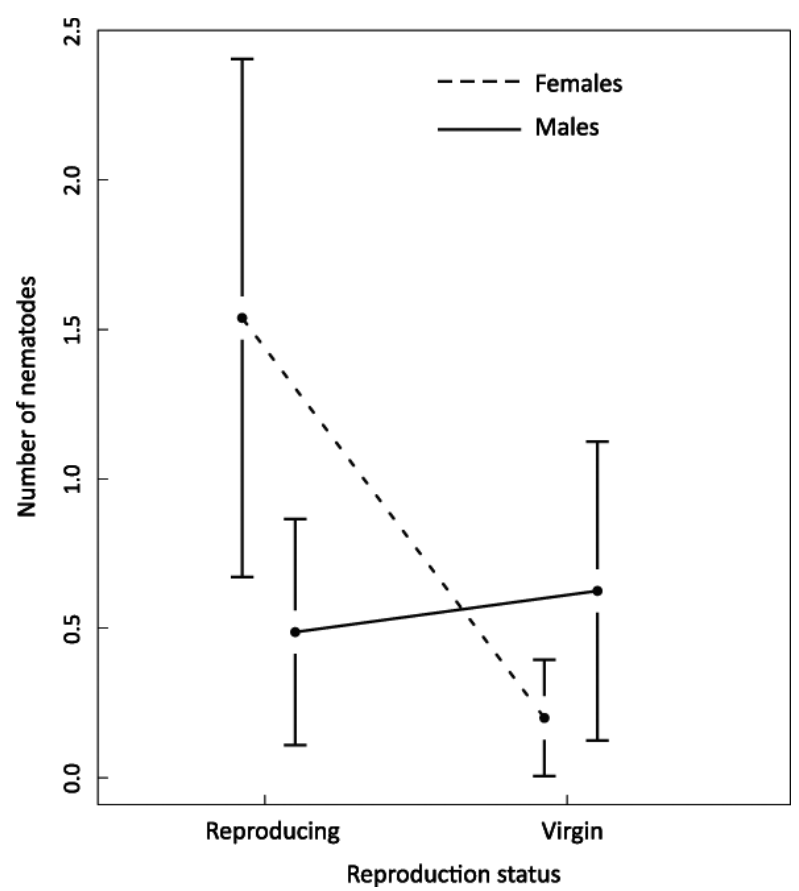

Fig. 1. The number of nematodes recorded in reproducing and virgin individuals of $T$. castaneum infected with $S$. feltiae. Mean and standard errors are shown.

min, so these values were used for further analyses. From each of four pseudo-replications of a given sample, the average phenoloxidase activity in units of absorbance was calculated. Changes in phenoloxidase activity were calculated as follows:

$$
P O_{a}=\frac{P O_{30}-P O_{5}}{25}
$$

where $P_{5}$ is phenoloxidase activity after $5 \mathrm{~min}, P O_{30}$ is phenoloxidase activity after $30 \mathrm{~min}$, and $P O_{a}$ is phenoloxidase activity in [units (of absorbance) $)^{-1 *} \mathrm{~min}^{-1}$ ].

\section{Protein assay}

The amount of protein was determined using the BCA (SigmaAldrich Co., St. Louis, MO, USA) method. A 10- $\mu$ l aliquot of each sample was mixed with $200 \mu 1$ of a $1: 50$ mixture of copper (II) sulphate and bicinchronic acid solution (Sigma-Aldrich), then incubated for $30 \mathrm{~min}$ at room temperature in darkness. Absorbance (wavelength $570 \mathrm{~nm}$ ) was measured. A standard curve was developed using a serial dilution of bovine serum albumin (BSA, Sigma-Aldrich, $4 \mathrm{mg} \mathrm{ml}^{-1}$ ). From this curve, the protein concentration in each sample was determined based on its absorbance.

\section{Normalization of phenoloxidase activity}

After averaging absorbance for all pseudo-replications of a given sample, values of phenoloxidase activity were normalized for protein content, using the following equation:

Table 1. Results of generalized linear models for number of IJ nematodes in $T$. castaneum beetles exposed to infection by $S$. feltiae (Poisson distribution). Factors marked in bold are statistically significant.

\begin{tabular}{|c|c|c|c|c|}
\hline Factor & Estimate & $\begin{array}{l}\text { Standard } \\
\text { Error }\end{array}$ & $z$ value & $p$ \\
\hline Interc & 0.232 & 0.255 & 0.910 & 0.363 \\
\hline Repro & 04 & 0.377 & -5.418 & $<0.001$ \\
\hline Sex & -1.15 & 0.263 & -4.37 & $<0.001$ \\
\hline Time & 0 & 0.007 & 0.921 & 0.357 \\
\hline Reproductive status $\times$ sex & 2.29 & 0.484 & 4.73 & $<0.001$ \\
\hline
\end{tabular}

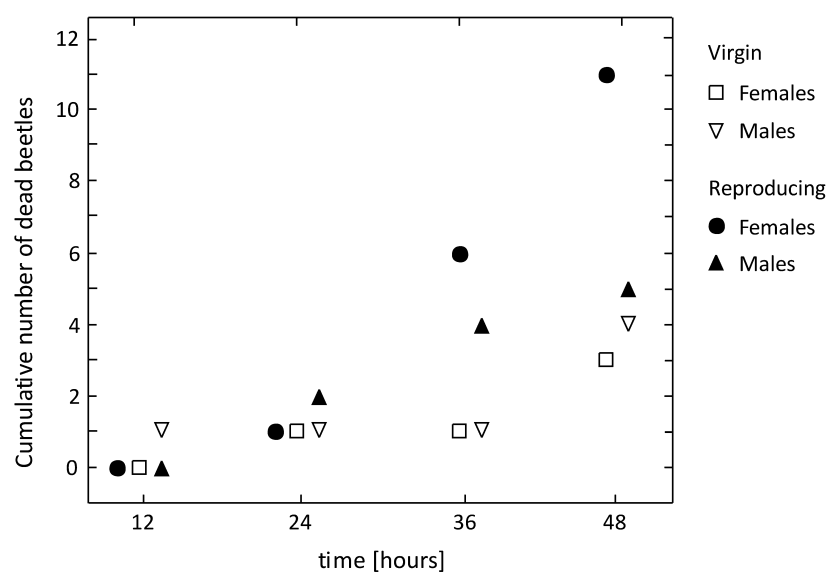

Fig. 2. The number of dead $T$. castenum recorded over time after infection with $S$. feltiae. No dead beetles were recorded in the control group (not exposed to nematodes) during the experiment; thus, the data presented are only for infected beetles. Cumulative number of dead beetles are shown.

$$
P O=\frac{P O_{a}}{P}
$$

where $P$ is protein concentration in mg and $P O$ is phenoloxidase activity in [units (of absorbance) ${ }^{-1 *} \mathrm{mg}$ protein ${ }^{-1 *} \mathrm{~min}^{-1}$ ].

\section{Statistical analyses}

The number of nematodes present was analyzed only for infected beetles. The tests used were dependent on the data distribution in each case: generalized linear models (GLM) for nematode number (Poisson distribution), GLM for survival (binomial distribution) and GLM for PO activity (Gaussian distribution). In the control group all animals survived. In the case of PO activity, data were $\log (\ln )$ transformed to obtain a normal distribution and body mass was used as a covariate. Statistical analyses were done using R software (R Development Core Team, 2012).

\section{RESULTS}

The number of nematodes infecting a beetle was small and depended on reproductive status $(\mathrm{p}<0.001)$ and sex $(\mathrm{p}$ $<0.001)$. We also detected significant interactions between sex and reproductive status $(\mathrm{p}<0.001)$, with reproducing females infected with the highest number of nematodes, whereas virgin males had a higher parasite load (Fig. 1; Table 1). Time had no effect on parasite load $(p=0.363)$. The factors that affected the proportion of dead beetles in the infected group were reproductive status $(\mathrm{p}=0.033)$, and time $(\mathrm{p}=0.0003)$, with mortality higher in reproducing animals and increasing with time (Fig. 2; Table 2).

In the case of PO activity, the interaction between infection and reproductive status was significant $(\mathrm{p}=0.007)$, but no main factor was statistically significant. Infected virgin

Table 2. Results of generalized linear models of the mortality of T. castaneum beetles exposed to infection by $S$. feltiae (binomial distribution). Factors marked in bold are statistically significant.

\begin{tabular}{lcccr}
\hline Factor & Estimate & $\begin{array}{c}\text { Standard } \\
\text { Error }\end{array}$ & z value & $p$ \\
\hline Intercept & 22.7 & 1292 & 0.018 & 0.986 \\
Infection & -18.8 & 1292 & -0.015 & 0.988 \\
Reproductive status & $\mathbf{1 . 1 0}$ & $\mathbf{0 . 5 1 6}$ & $\mathbf{2 . 1 4}$ & $\mathbf{0 . 0 3 3}$ \\
Sex & 0.602 & 0.498 & 1.21 & 0.227 \\
Time & $\mathbf{- 0 . 0 8 1}$ & $\mathbf{0 . 0 2 2}$ & $\mathbf{- 3 . 5 9}$ & $<\mathbf{0 . 0 0 1}$ \\
\hline
\end{tabular}




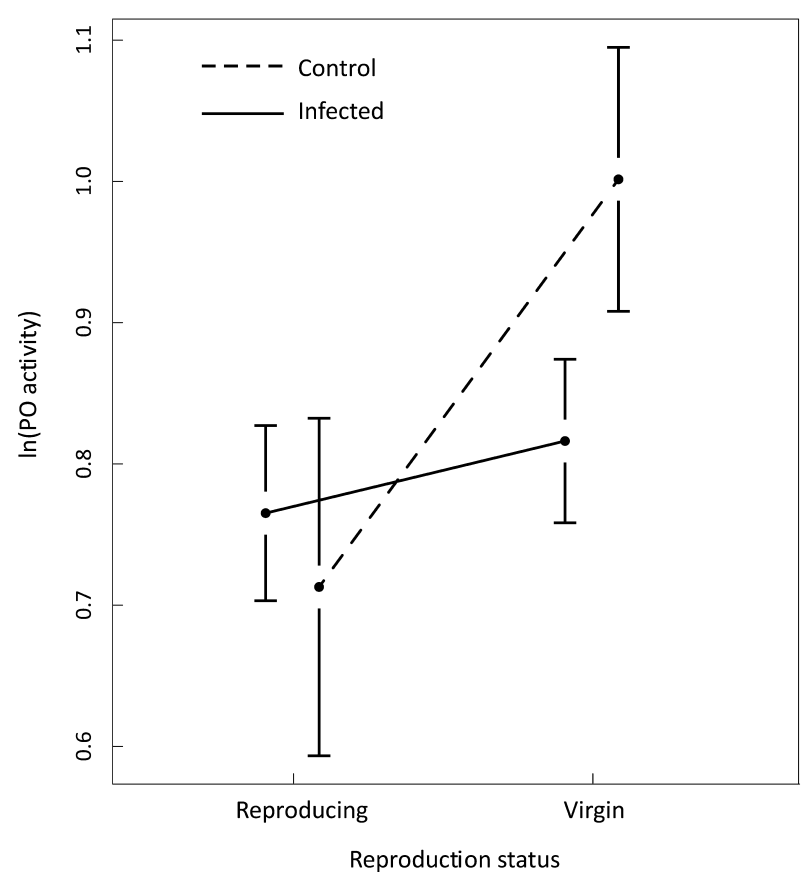

Fig. 3. The phenoloxidase (PO) activity (units of absorbance ${ }^{-1 *} \mathrm{mg}$ protein ${ }^{-1 *} \mathrm{~min}^{-1}$ ) recorded in reproducing and virgin $T$. castanuem following infection with $S$. feltiae. Mean and standard errors are shown.

beetles had the highest PO activity, but infection decreased PO activity in reproducing animals (Fig. 3, Table 3).

\section{DISCUSSION}

Our study confirmed that reproduction may impair the activity of phenoloxidase, as is stated by Rolff \& SivaJothy (2002). We recorded the highest activity in virgin beetles and the lowest in reproducing animals, indicating that reproduction decreased the amount of resources available for the immunological response. Likewise, virgin females contained the lowest number of nematodes of all the treatment groups. At the same time, reproducing beetles infected with $S$. feltiae suffered a higher mortality than virgins. Our results thus seem to indicate that reproduction incurs costs that can decrease the effectiveness of the immunological response, while also increasing the cost of that response in reproducing individuals.

This finding seems to confirm the existence of a trade-off between reproductive activity and immune activity. At the same time, however, we did not find strong support for the assumptions of Bateman's Principle, which states that it is

Table 3. Results of generalized linear models of the phenoloxidase (PO) activity recorded for $T$. castaneum beetles exposed to infection by S. feltiae (normal distribution). Data were log (In) transformed. Factors marked in bold are statistically significant.

\begin{tabular}{|c|c|c|c|c|}
\hline Factor & Estimate & $\begin{array}{c}\text { Standard } \\
\text { Error }\end{array}$ & $\mathrm{d}_{\mathrm{t} \text { value }}$ & $\mathrm{p}$ \\
\hline Intercept & 0.674 & 0.068 & 9.88 & $<0.001$ \\
\hline Infection & -0.052 & 0.061 & -0.857 & 0.392 \\
\hline Reproductive status & 0.051 & 0.060 & 0.844 & 0.400 \\
\hline Sex & 0.057 & 0.043 & 1.33 & 0.186 \\
\hline Time & 0.002 & 0.002 & 1.28 & 0.202 \\
\hline Reproductive status $\times$ infection & 0.238 & 0.086 & 2.75 & 0.006 \\
\hline
\end{tabular}

the female sex that should invest more in parasite resistance (Bateman, 1948; Rolff, 2002). There are many studies on invertebrates in which males are more susceptible to parasites. For example, Gray (1998) reports a higher mortality in male house crickets (Acheta domestica, L. 1758) after experimental infection with Serratia liquefaciens and, similarly, Wedekind \& Jakobsen (1998) show that male copepods, Macrocyclopus albidus (Jurine, 1820), are more likely to be infected by helminths. Likewise, Adamo et al. (2001) demonstrate a similar effect in Gryllus texensis exposed to $S$. marcescens.

At first, the results of our phenoloxidase assays also seem to conflict with many reports in the literature: we did not record differences in phenoloxidase activity between the sexes. There are a number of studies on sexual dimorphism in the immune response (specifically, phenoloxidase activity), most of which record a significantly higher phenoloxidase activity in females. For example, a meta-analysis by Nunn et al. (2009) includes 11 studies on phenoloxidase, of which 10 report female-biased phenoloxidase and only one a male bias. Likewise, our results also conflict with the existing literature in reporting a reproduction-based difference in phenoloxidase activity, as there are also studies that report no such differences between groups of different reproductive status. For example, Schwarzenbach et al. (2005) report that sexes of the yellow dung fly (Scathophaga stercoraria, L. 1758) differ in hemolymph phenoloxidase activity only significantly associated with age, not copulation or egg laying.

An explanation for this may be found using optimal resource allocation models. As the resources available to an organism are limited, they should be invested in a way that maximizes fitness (so-called "Allocation Principle" see: Perrin \& Sibly, 1993). Their allocation to reproduction will decrease the amount available for maintenance (including immunological defence) (Cichoń, 1997). However, neither reproduction nor the immune response are simple processes. Reproduction imposes costs at every stage of reproductive activity (that is, gamete production, courting, mating, guarding and parental care) and all of these may also pose a significant cost for males (see, for example, Scharf et al., 2012). Likewise, an insect's immunological system consists of a range of mechanisms from humoral (for example, production of reactive oxygen species and antibacterial peptides) to cellular (such as encapsulation and phagocytosis) (Gillespie et al., 1997; Lavine \& Strand, 2002). It is the interplay between all these elements, mediated by the mating system and the environment, which shapes the reproduction-based differences between sexes in their susceptibility to parasite infection. For example, Stoehr \& Kokko (2006) propose that as the strength of sexual selection on males increases, so too should the magnitude of the sex-based difference in immune response. However, in exceptional cases males may still invest the same amount of resources as females, or even more, in the immune response, depending on the effect of parasites on condition and/or on the relationship between condition and reproduction (Stoehr \& Kokko, 2006). 
We therefore propose that the lack of a difference in mortality between the sexes may be the result of decreased sexual selection on the males in our laboratory culture; under these circumstances, both sexes may eventually invest similar amounts of resources in their immune response. At the same time, the distribution of the costs of the different stages of reproductive activity and investment in particular parts of the immune system may differ greatly between the sexes, which may be the cause of the recorded patterns in the particular traits investigated. One should also keep in mind that we checked mortality only after $48 \mathrm{~h}$. As $T$. castaneum is a long lived animal, differences in survival between sexes might only become apparent after a longer period of time.

Our results indicate that using virgin instead of mated animals is not recommended, as the dynamics of infection and mortality may be influenced by the costs of reproduction. Results that are generated with virgin individuals are unlikely to resemble real-world phenomena, particularly if the study organism is a highly promiscuous species and is likely to experience significant costs of reproduction throughout its adult life. Additionally, we have shown that prolonged exposure to $S$. feltiae may be lethal even for adult $T$. castaneum despite the fact that they are not the primary infection targets for this commercially used entomopathogenic nematode.

ACKNOWLEDGEMENTS. We thank L. Higgins for the editorial correction and useful insights. We also thank J. Ziemba and A. Ziobro for their assistance in the laboratory and two anonymous reviewers for their remarks on an earlier version of this manuscript. The project was supported by the Polish National Science Centre (grant no. NCN 2011/01/B/NZ8/00105) and Jagiellonian University (DS/WBINOZ/INOŚ/747). The funding source had no involvement in study design, collection, analysis and interpretation of data

COMPETING INTERESTS. The authors declare no competing financial interests. P.K. developed the concept and designed the research, D.M and M.G. performed the experiment, S.M.D. did statistical analyses, D.M., P.K. and J.H. wrote the paper, J.H. designed phenoloxidase measurements.

\section{REFERENCES}

Adamo S.A., Jensen M. \& Younger M. 2001: Changes in lifetime immunocompetence in male and female Gryllus texensis (formerly $G$. integer): trade-offs between immunity and reproduction. - Anim. Behav. 62: 417-425.

Alexander J. \& Stimson W.H. 1988: Sex hormones and the course of parasite infection. - Parasitol. Today 4: 189-193.

Bateman A.J. 1948: Intra-sexual selection in Drosophila. - Heredity 2: 349-368.

BAtHon H. 1996: Impact of entomopathogenic nematodes on non-target hosts. - Biocontr. Sci. Techn. 6: 421-434.

Bucher G. 2009: The Beetle Book. Published online by the author. URL: www.gwdg.de/ gbucher1/tribolium-castaneumbeetle-book1.pdf.

CicHoN M. 1997: Evolution of longevity through optimal resource allocation. - Proc. R. Soc. Lond. (B) 264: 1383-1388.

Cerenius L., Lee B.L. \& Söderhäll K. 2008: The proPO-system: pros and cons for its role in invertebrate immunity. - Trends Immunol. 29: 263-271.
Córdoba-Aguilar A. \& Munguía-Steyer R. 2013: The sicker sex: understanding male biases in parasitic infection, resource allocation and fitness. - PLOS ONE 8(3): e76246, 7 pp.

Córdoba-Aguilar A., Ruiz-Silva D., Munguía-Steyer R. \& Lanz-Mendoza H. 2011: Do reproductive activities compromise immunological competence as measured by phenoloxidase activity? Field and experimental manipulation in females of two damselfly species. - Physiol. Entomol. 36: 335-342.

Cotter S.C., KRUUK L.E.B. \& Wilson K. 2004: Costs of resistance: genetic correlations and potential trade-offs in an insect immune system. - J. Evol. Biol. 172: 421-429.

Freitak D., Knorr E., Vogel H. \& Vilcinskas A. 2012: Genderand microRNA expression in Tribolium castaneum. - Biol. Lett. 8: 860-863.

French S.S., De Nardo D.F. \& Moore M.C. 2007: Trade-offs between the reproductive and immune systems: facultative responses to resources or obligate responses to reproduction? Am. Nat. 170: 79-89.

Georgis R., Koppenhofer A.M., Lacey L.A., Bélair G., Duncan L.W., Grewal P.S., SAmish M., Tan L., Torr P. \& van Tol R.W.H.M. 2006: Successes and failures in the use of parasitic nematodes for pest control. - Biol. Contr. 38: 103-123.

Gillespie J.P., Kanost M.R. \& TrenczeK T. 1997: Biological mediators of insect immunity. - Annu. Rev. Entomol. 42: 611643.

GonzÁlez-Santoyo I. \& Córdoba-Aguilar A. 2012: Phenoloxidase: a key component of the insect immune system. - Entomol. Exp. Appl. 142: 1-16.

GRAY D.A. 1998: Sex differences in susceptibility of house crickets, Acheta domesticus, to experimental infection with Serratia liquefaciens. - J. Invertebr. Pathol. 71: 288-289.

Hirao A., Ehlers R.-U. \& Strauch O. 2010: Life cycle and population development of the entomopathogenic nematodes Steinernema carpocapsae and S. feltiae (Nematoda, Rhabditida) in monoxenic liquid culture. - Nematology 12: 201-210.

KraAiJeveld A. \& Godfray H. 1997: Trade-off between parasitoid resistance and larval competitive ability in Drosophila melanogaster. - Nature 389: 278-280.

LAVINE M.D. \& STRAND M.R. 2002: Insect haemocytes and their role in immunity. - Insect Biochem. Mol. Biol. 32: 1295-1309.

Milutinović B., Stolpe C., Peub R., Armitage S.A. \& Kurtz J. 2013: The red flour beetle as a model for bacterial oral infections. - PloS ONE 8(5): e64638, 12 pp.

Moore S.L. \& WiLson K. 2002: Parasites as a viability cost of sexual selection in natural populations of mammals. - Science 297: 2015-2018.

Nava-Sánchez A., González-Tokman D., Munguía-Steyer R. \& Córdoba-Aguilar A. 2015: Does mating activity impair phagocytosis-mediated priming immune response? A test using the house cricket, Acheta domesticus. - Acta Ethol. 18: 295-299.

Nunn C.L., Lindenfors P., Pursall E.R. \& Rolff J. 2009: On sexual dimorphism in immune function. - Philos. Trans. $R$. Soc. Lond. (B) 364: 61-69.

Perrin N. \& Sibly R. 1993: Dynamic models of energy allocation and investment. - Annu. Rev. Ecol. Syst. 24: 379-410.

Poulin R. 1996: Sexual inequalities in helminth infections: a cost of being a male? - Am. Nat. 147: 287-295.

R Development Core Team 2012: R: A Language and Environment for Statistical Computing. R Foundation for Statistical Computing, Vienna, Austria.

Ramos-Rodríguez O., Campbell J.F. \& Ramaswamy S.B. 2006: Pathogenicity of three species of entomopathogenic nematodes to some major stored-product insect pests. - J. Stor. Prod. Res. 42: 241-252. 
Rolff J. 2002: Bateman's principle and immunity. - Proc. $R$. Soc. (B) 269: 867-872.

RolfF J. \& Siva-Jothy M.T. 2002: Copulation corrupts immunity: a mechanism for a cost of mating in insects. - Proc. Natl. Acad. Sci. U.S.A. 99: 9916-9918.

Rossi E., Cosimi S. \& Loni A. 2010: Insecticide resistance in Italian populations of Tribolium flour beetles. - Bull. Insectol. 63: $251-258$

Scharf I., Peter F. \& Martin O.Y. 2012: Reproductive tradeoffs and direct costs for males in arthropods. - Evol. Biol. 40: 169-184.

Schmid-Hempel P. 2011: Evolutionary Parasitology: The Integrated Study of Infections, Immunology, Ecology, and Genetics. Oxford University Press, New York, 544 pp.

Schmid-Hempel P. \& EBert D. 2003: On the evolutionary ecology of specific immune defence. - Trends Ecol. Evol. 18: 27-32.

Schwarzenbach G.A., Hosken D.J. \& Ward P.I. 2005: Sex and immunity in the yellow dung fly Scathophaga stercoraria. J. Evol. Biol. 18: 455-463.

Siva-Jothy M.T., Tsubaki Y. \& Hooper R.E. 1998: Decreased immune response as a proximate cost of copulation and oviposition in a damselfly. — Physiol. Entomol. 23: 274-277.
Siva-Jothy M.T., Tsubaki Y., Hooper R.E. \& Plaistow S.J. 2001: Investment in immune function under chronic and acute immune challenge in an insect. - Physiol. Entomol. 26: 1-5.

SокоLOFF A. 1974: The Biology of Tribolium, with Special Emphasis on Genetic Aspects. Vol. 2. Clarendon Press, Oxford, $610 \mathrm{pp}$.

Stoenr A.M. \& Koкко H. 2006: Sexual dimorphism in immunocompetence: what does life-history theory predict? - Behav. Ecol. 17: 751-756.

WeDEKIND C. \& JAKOBSEN P.J. 1998: Male-biased susceptibility to helminth infection: an experimental test with a copepod. Oikos 81: 458-462.

ZuK M. 1990: Reproductive strategies and disease susceptibility: an evolutionary viewpoint. — Parasitol. Today 6: 231-233.

ZuK M. \& McKean K.A. 1996: Sex differences in parasite infections: patterns and processes. - Int. J. Parasitol. 26: 1009 1024.

Received November 25, 2015; revised and accepted March 14, 2016 Published online April 5, 2016 